Ocean Bottom Seismic - the Original Broadband - How Nodal Technology and Blended Sources Make it Cost Effective

C. Walker* (Fairfield Nodal), D. Hays (Fairfield Nodal) \& S. McIntosh (Fairfield Nodal)

\title{
Introduction
}

Broadband marine is not new - the original method for broadband marine data acquisition by combining vector (vertical geophone) and scalar (hydrophone) data was proposed by Milo Backus in 1958! (Water Reverberations--Their Nature and Elimination, Geophysics, 1958)

This ocean bottom dual sensor approach has been applied, primarily for appraisal and development applications, for a many years worldwide but the scale of the surveys, by dint of their focus on field specific imaging objectives, has been limited compared to towed streamer surveys in both size and duration. One of the challenges set by the oil companies has been to reduce the unit costs of ocean bottom data - "If only the square kilometer rates were lower we would shoot more data" is a common mantra.

The difficulty in doing this has been the inherent technical downtime experienced by all the contractors operating ocean bottom systems - the terminations, connectors, power distribution and data telemetry components within a traditional ocean bottom cable (OBC) system are inherently prone to failure due to the intrinsic nature of the cable deployment/recovery cycle where the cables are stressed and de-stressed every time they are laid onto/recovered from the seabed. It is akin to recovering and deploying the full streamer spread every line change for towed streamer operations.

\section{Nodal Technology}

The intrinsic reliability of battery powered autonomous seismic recording units - nodes - allows ever larger spreads to be operated, unlike traditional cable-based systems where technical downtime and the need to connect the active spread to either a central recording vessel or a series of recording buoys limits the size of the live spread. This "bigger paintbrush" results in reduced shot effort since duplicate shots, the Achilles heel of ocean bottom seismic (OBS) surveys, can be reduced and in some cases eliminated. Further the removal of fixed interval receiver stations - determined during the manufacture of OBC systems - allows for increased receiver intervals to be employed which again increases the areal size of the active spread.

\section{Blended Sources}

Looking at the very dramatic improvements in both data quality and operational productivity that have been achieved onshore using a variety of "simultaneous" vibratory sources, the uplift in OBS survey performance that can be achieved by firing more than one source into an OBS spread drops straight to the bottom line since survey durations can be almost cut in half for a relatively modest increase in daily operating cost resulting from the addition of another source vessel.

The key to this, of course, is the ability to separate or "de-blend" the two (or more) shots recorded by each receiver. We have adopted the approach taken by Delft University (Mahdad, A., et. al., 2011, Separation of blended data by iterative estimation and subtraction of blending interference noise: Geophysics,76, Q9-Q17) namely to remove from the desired source those incoherent events from the other or un-desired source. Rather than try to synchronize the sources with a defined intra-source time dither as has been done for towed streamer blended source acquisition (Beasley, et. al., SEG Abstracts, 2012) we have adopted an autonomous source approach, proposed and patented by Apache Corporation, whereby each source vessel operates entirely independently of the other firing its source at pre-determined pseudo-random locations. Consequently the energy from the "other" shots appears as random events in the common receiver gathers for the "desired" shots, as illustrated in Figure 2. Figure 1 shows the common shot gathers before and after de-blending for the data extracted from the continuous data recorded in the nodes according to shot time for one of the two shooting vessels. 


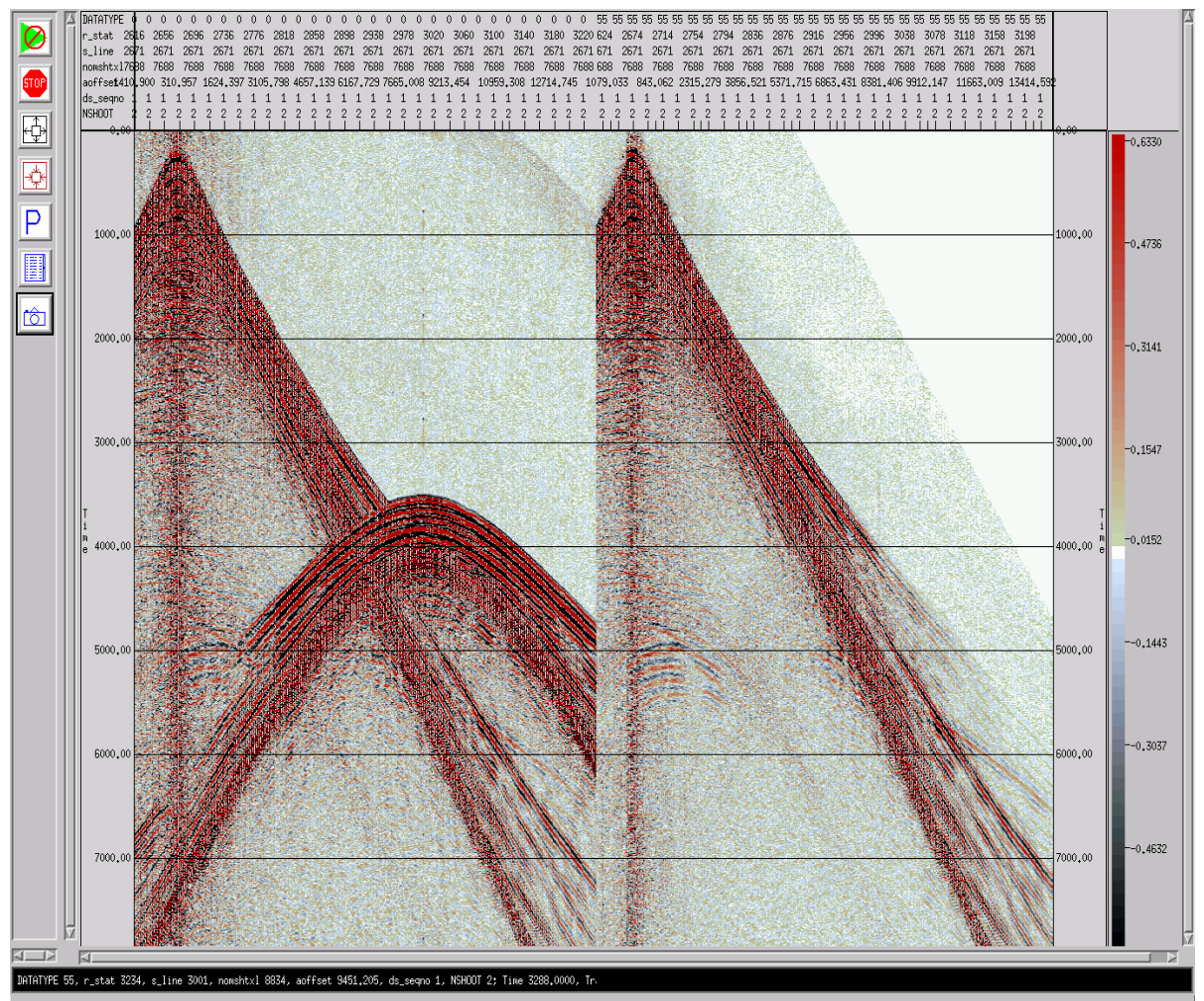

Blended Shot

De-Blended Shot

Figure 1 Common Shot Gathers - beforelafter de-blending.

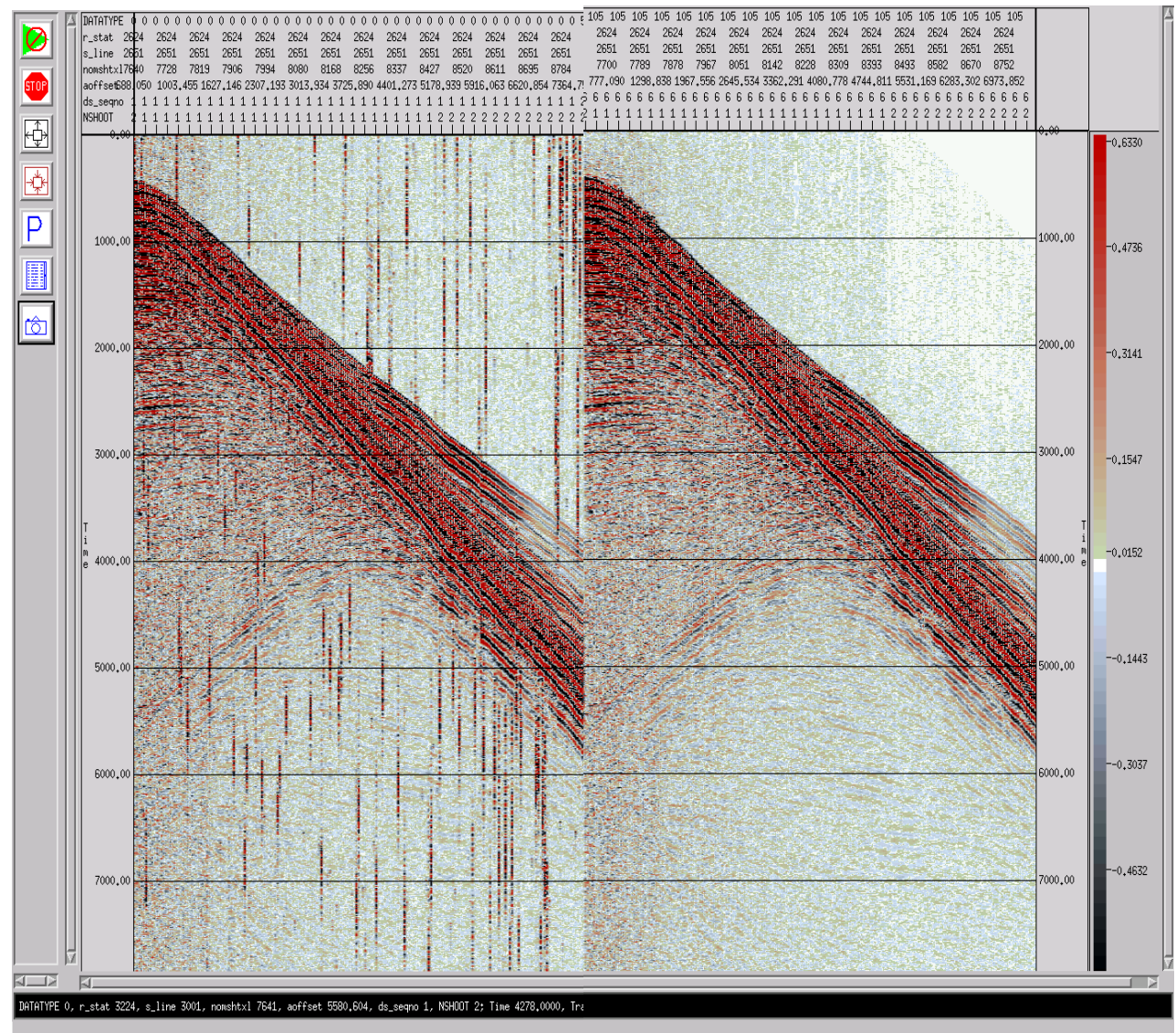

Blended Receiver De-Blended Receiver

Figure 2 Common Receiver Gathers - beforelafter de-blending. 


\section{Economic Impact}

By removing the traditional sources of ocean bottom technical downtime through the utilisation of node technology and successfully applying the latest developments in the use of blended sources, the historical economic barrier to using ocean bottom seismic data for larger scale projects no longer applies so it is expected that there will be a wider application of nodal blended source surveys in the future. 